\title{
Pushing the XEDS Boundaries in Materials Research: Low Voltage XED Spectrum Imaging in the FEG-SEM
}

\author{
Arne Janssen $^{1}$, M. G. Burke ${ }^{1}$, and Simon Burgess ${ }^{3}$ \\ ${ }^{1}$ Materials Performance Centre, School of Materials, The University of Manchester, Manchester, UK. \\ ${ }^{2}$ Oxford Instruments NanoAnalysis, UK
}

Low-voltage high resolution imaging in advanced field emission gun scanning electron microscopes (FEG-SEMs) is an essential technique in the characterisation of a broad range of materials - particularly for polymers and complex multiphase/multicomponent materials, that can experience significant charging effects during conventional 10 to $20 \mathrm{kV}$ imaging. In particular, the analysis of corrosion specimens can be problematic due to electron charging effects when studying the fine structure of oxides. Previously, it was necessary to change FEG-SEM operating conditions to generate x-ray energy dispersive spectroscopy (XEDS) data. This could make it difficult to locate the same feature under significantly different imaging conditions, and is particularly challenging for beam-charging materials. In this study we have applied low voltage XEDS analysis to evaluate an austenitic stainless steel corrosion specimen to study the oxides in cracks.

For the study, a Schottky-type FEG-SEM, Zeiss Merlin with Gemini II column and equipped with a Oxford Instruments X-Max ${ }^{\mathrm{N}} 150$ silicon drift detector (SDD) and an X-Max Extreme windowless SDD for XED spectrum imaging and analysis were used. All SEM images were obtained with a parallel onaxis in-lens secondary electron (SE) detector with primary electron beam energy of $1.5 \mathrm{kV}, 3 \mathrm{kV}, 5 \mathrm{kV}$ and $15 \mathrm{kV}$, respectively. During XEDS acquisition, the SEM condition were unchanged expect for the accelerating voltage and the working distance. XED spectrum images were acquired with the Oxford Instruments AZtecEnergy acquisition and XEDS analysis software with a spectrum image resolution of 512 x 512 pixel and a pixel dwell time of $1000 \mu \mathrm{s}$. All spectrum images were processed using the Oxford Instruments TruMap processing algorithms for peak deconvolution and background subtraction. No image enhancement was used for these spectrum images (no enhanced contrast or brightness).

The region of interest in this sample was a small oxide-filled crack in a metallographic sample, polished to a $1 \mu \mathrm{m}$ diamond finish. Figure 1 compares all SE images as a function of accelerating voltage, with a notable improvement in the morphology of the oxide of the SE image for low voltages. Each row in the figure provides the SE image as well as the XED spectrum images for O (K), Fe (L) and Ni (L). The detail revealed in the Fe spectrum images acquired at $1.5 \mathrm{kV}$ and $3 \mathrm{kV}$ clearly show the presence of a 2layer oxide structure: an Fe-poor oxide adjacent to the oxide/metal interface, whereas these details are absent for the $15 \mathrm{kV}$ spectrum image. In this particular corrosion specimen, fine Ni-rich features were detected within the oxide-filled crack. The details in the Ni L spectrum images at $1.5 \mathrm{kV}$ and $3 \mathrm{kV}$ are striking. The ability to generate viable XEDS datasets at low voltages provides significant improvements in spatial resolution of the analysis due to the significantly reduced depth of $\mathrm{x}$-ray generation in the sample. Thus, low voltage XEDS is providing new insights into materials analysis and new options for microstructural characterization. [1]

\section{References:}

[1] We thank James Holland for valuable technical discussions and suggestions. 

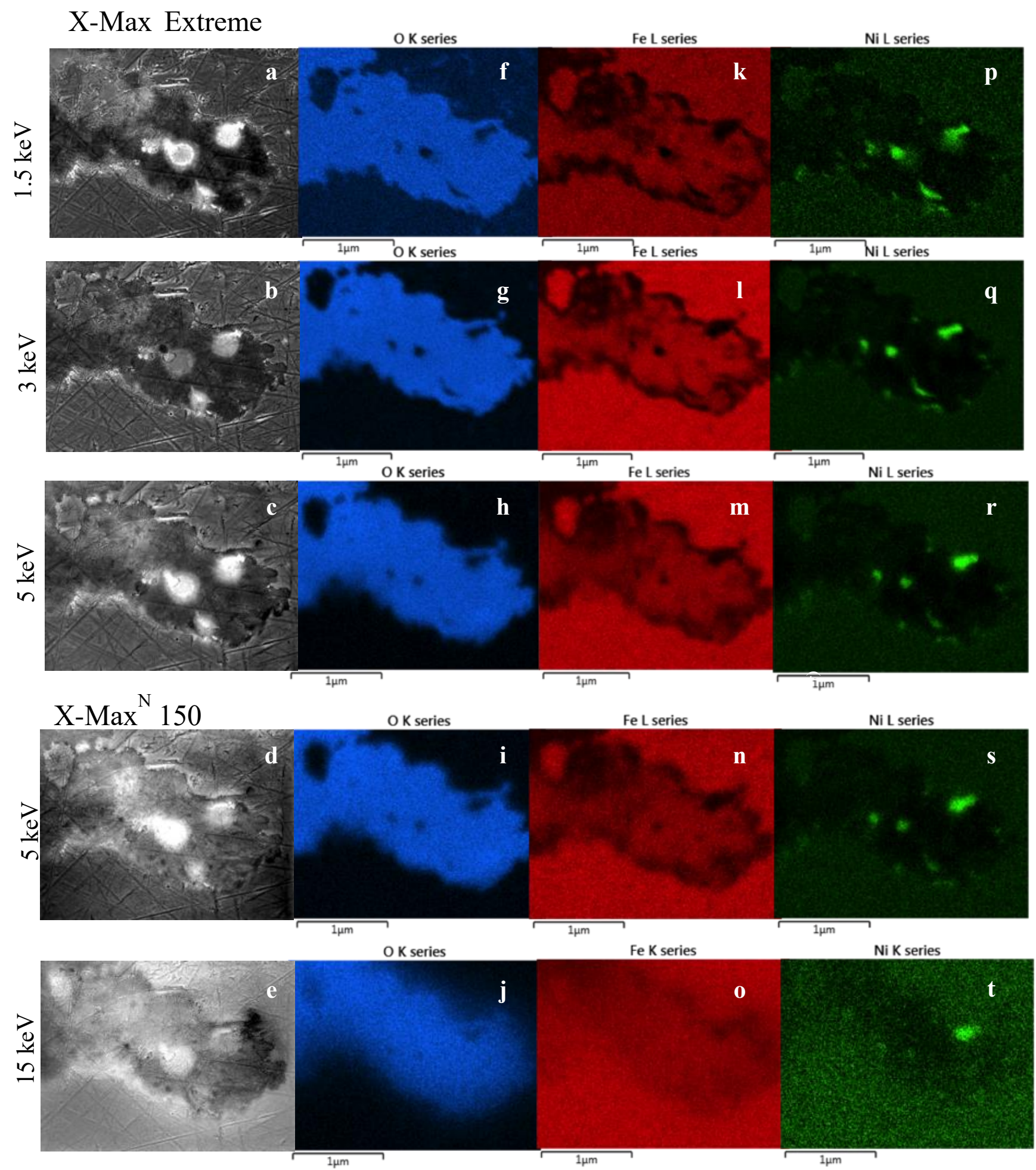

Figure 1. SE images observed with primary electron beam energy of $1.5 \mathrm{keV}$ (a), $3 \mathrm{keV}$ (b), $5 \mathrm{keV}$ (c-d) and $15 \mathrm{keV}$ (e). Comparison of spatial the resolution of XED spectrum images for X-Max Extreme and $\mathrm{X}_{-\mathrm{Max}^{\mathrm{N}}} 150$ for O-K series (f-h)-(i,j), Fe-L series (k-m)-(n,o) and Ni-L series (p-r)-(s-t) at selected accelerating voltages. 